\title{
Osg4Web. Condivisione di dati e applicazioni VR WebGIS per il paesaggio archeologico
}

\author{
Luigi Calori ${ }^{\dagger}$, Carlo Camporesi ${ }^{\ddagger}$, \\ Augusto Palombini ${ }^{\ddagger}$, Sofia Pescarin ${ }^{\ddagger}$
}

\begin{abstract}
Sommario. Perché ricostruire il paesaggio archeologico? Cosa possiamo effettivamente ricostruire? Quale tecnologia e quale approccio epistemologico, se ne esiste uno, può contribuire ad ottenere migliori risultati nel campo della ricerca? E infine perché utilizzare la rete come mezzo di comunicazione, scambio e lavoro sul paesaggio antico?

AвSTRACT. Why trying to reconstruct archaeological landscape? What can we really reconstruct? Which kind of technologies and which theoretical approach, if any, can help us in obtaining the best research results? And why using the internet as communicating, exchanging and workinf tool for the ancient landscape? The paper approaches such problems through the illustration of the Osg4Web tool for archaeological landscape data sharing.
\end{abstract}

Ciò che osserviamo oggi è che il lento processo di scomparsa delle tracce archeologiche sul territorio sta accelerando, in maniera spesso irreversibile. Il rischio di perdere completamente interi patrimoni storici e archeologici è tangibile, e con essi anche la memoria storica di intere collettività. Ciò che dovrebbe essere trattato come patrimonio dell'uomo viene invece spesso gestito come proprietà privata, anche dagli stessi gruppi di lavoro che dovrebbero studiarlo. Il risultato a livello globale sembra condurre ad uno sviluppo 
sempre più lento e insignificante della ricerca. I risultati rimangono spesso non pubblicati o appannaggio di pochi eletti. La pratica dello scambio delle informazioni e dei dati, anche all'interno degli stessi gruppi di ricerca, risulta spesso non perseguita, così come la comunicazione all'esterno di ciò che si fa. I processi di validazione di tipo peer-review sono carenti, anche a causa di una mancata trasparenza dei risultati e dei metodi scientifici utilizzati. E' possibile però tentare di modificare questa tendenza, anche attraverso l'utilizzo di strumenti e approcci aperti e della rete come strumento di lavoro condiviso, prima ancora che di comunicazione.

Il Virtual Heritage Lab dell'Istituto per le Tecnologie applicate ai Beni Culturali, del CNR (CNR ITABC), lavora ormai da anni su progetti di valorizzazione del patrimonio culturale attraverso due canali preferenziali corrispondenti a due filiere di elaborazione distinte: le applicazioni di realtà virtuale e la restituzione di piatteforme GIS tridimensionali per la consultazione e la navigazione via web.

L'approccio di tipo Open che è stato in questo senso portato avanti ha un duplice risvolto: quello sugli strumenti e quello sui dati. Il primo viene intenso come ricerca finalizzata a utilizzare sempre più spesso software Open Source, il secondo rappresenta l'obiettivo di rendere quanto più possibile trasparente l'elaborazione e la presentazione delle informazioni. Tale tentativo si concretizza, ad esempio, attraverso l'attribuzione a ciascun elemento (layer, oggetto VR) di metadati riguardanti fonti, metodologie, software, autori (e disponibilità di download ove possibile). E' indispensabile infatti secondo il nostro approccio mantenere distinti i concetti di efficacia ricostruttiva e attendibilità del dato, due elementi entrambi di elevata importanza ma che è evidentemente bene trattare su piani separati (Camporesi, Palombini e A. Pescarin 2007), anche ad evitare sterili e frequenti polemiche su quella spettacolarizzazione dell'archeologia cui non corrisponderebbe rigorosità scientifica.

Recentemente i 3D WebGIS stanno registrando un notevole successo (S. Pescarin e Calori 2005), ciò grazie anche alla diffusione di applicazioni come Google Earth (http://earth.google.com/). Con il termine 3D WebGIS comunemente si definiscono i GIS 3D, accessibili attraverso il Web, e che consentono la visualizzazione di modelli digitali del terreno tridimensionali fruibili in maniera dinamica tramite un client, al quale il server su cui risiedono le informazioni - DTM, fotografie aeree o da satellite, layer tematici, livelli di dettaglio - invia le informazioni necessarie alla visualizzazione, a seconda delle interazioni dell'utente. Il tipo di applicazioni su cui il VHLab sta lavorando da qualche anno (Calori, Camporesi, Palombini et al. 2006; Pescarin S. 2006) è stato denominato "VR webGIS" in parte anche per differenziarsi dai più tradizionali webGIS tridimensionali. La componente che contraddistingue il sistema dal lato utente è infatti una forte interattività e immersività nel paesaggio archeologico, elementi, questi, che lo avvicinano più alle applicazioni scientifiche di Realtà Virtuale o ai giochi on line che ai tradizionali GIS. Dei Sistemi Informativi Geografici conservano invece la componente spaziale dei dati. Questo 
elemento è fondamentale perché consente di utilizzare un unico processo di elaborazioni a partire dal campo fino al web, costruendo delle basi di dati geospaziali comuni a diverse applicazioni (on-line e off-line), aggiornabili.

La filiera di lavoro riguardante la parte VR WebGIS si compone essenzialmente di quattro passaggi, caratterizzati principalmente da software Open Source (Calori, Camporesi, Forte et al. 2005).

(1) La realizzazione del progetto GIS e del modello digitale del terreno (GRASS-GIS)

(2) La creazione di paesaggi virtuali (OSGdem)

(3) La manipolazione dei paesaggi e la loro pubblicazione (Visual Nature Studio, Virtual Terrain Project: Enviro Xtend)

(4) La fruizione interattiva attraverso il web (Osg4Web plug-in di interfacciamento con il web browser)

Il primo passaggio consiste nella vera e propria definizione e composizione dei dati geografici, l'elaborazione del modello digitale di terreno e lo sviluppo del progetto GIS complessivo (operazioni effettuate con GRASS-GIS). La seconda prevede la traduzione del modello di terreno in un modello paginato e adatto alla trasmissione di dati via web (compito svolto da OSG), e nella creazione del paesaggio virtuale.

Nella terza fase il paesaggio viene arricchito sia dei layer informativi GIS che degli elementi tridimensionali (alberi, edifici, elementi naturali, etc.) che caratterizzano una visione 3D. Questa fase è gestita dal pacchetto VTP, di cui si parlerà più diffusamente, con la recente aggiunta dell'unico software commerciale citato in questa catena, Visual Nature Studio, che presenta caratteristiche assolutamente peculiari per quanto riguarda la capacità di creazione e gestione di ecosistemi, nonché la compatibilità in input/output con una vastissima gamma di formati open e proprietari.

Infine, la pubblicazione sul web, prevede la successiva gestione da parte dell'utente tramite un plug-in per i comuni internet browser (OSG4Web) che rappresenta il principale risultato degli ultimi mesi di attività del VHLab.

OpenSceneGraph (OSG: http://www.openscenegraph.org/) è una libreria Open Source per la generazione di applicativi di visualizzazione 3D (Kuehne e Martz 2007). La sua caratteristica fondamentale è la struttura basata su OpenGL e totalmente Cross-Platform (Windows, Linux, MacOS, ...). OSG permette la gestione di dati geografici e gestisce la paginazione di modelli territoriali, permette inoltre il reperimento di modelli 3D tramite internet.

Il pacchetto VTP (Virtual Terrain Project: http://www.vterrain.org/) è composto da diversi applicativi per la gestione, il trattamento e la visualizzazione interattiva di dati GIS. Nella versione originale consta di tre elementi:

VTBuilder: Software per la visualizzazione e la preparazione di dati GIS canonici 
CManager: Permette la visualizzazione di modelli 3D e la creazione di librerie

Enviro: Crea paesaggi 3D da dati geografici navigabili in Real-Time. Permette la modifica dei paesaggi direttamente dalla visualizzazione 3D

La versione Enviro X-tend realizzata dal VHLab, in collaborazione con il CINECA, permette la visualizzazione di terreni generati tramite OSGdem (OSG) all'interno della scena e prevede la possibilità di salvataggio delle strutture di Enviro sul terreno esterno.

Il punto focale del lavoro del VHLab nell'ottica del VR WebGis è comunque il plug-in OSG4Web, che rappresenta il traguardo realmente inedito dell'ultimo anno di lavoro. OSG4Web è un framework di sviluppo Open Source per la creazione di applicativi di visualizzazione 3D realtime attraverso il Web (Akenine-Möller e Haines 2002). Produce componenti aggiuntivi per Internet Explorer e Mozilla Firefox. E' interamente scritto in C++ e lo Standard Core di visualizzazione si basa sulla libreria OpenSceneGraph.

OSG4Web è un sistema in prospettiva multi piattaforma. Attualmente sono disponibili porting per: Windows (versione 1.0.2.1) - Linux, MacOS in debug. Lo Standard Core (core nativo rilasciato con il codice sorgente di OSG4Web) è specifico per la visualizzazione e di paesaggi archeologici e l'interazione con dati geografici.

Le caratteristiche principali di Osg4Web sono la visualizzazione di terreni paginati e modelli complessi attraverso il web, il caricamento delle tile del terreno on demand e lo switching durante il processo di rendering, l'aggiunta $e$ lo switching di layer vettoriali 3D, l'aggiunta e lo switching di label statiche e scalabili, la creazione della scena e l'aggiunta di modelli 3D esterni sul terreno tramite funzioni di scripting (ibidem).

A queste si aggiungono recenti implementazioni quali: l'orientamento nella scena attraverso mappa 2D, una funzione JavaScript FlyTo per il posizionamento immediato su coordinate predefinite, vari strumenti di navigazione ed interazione con la scena selezionabili dall'interfaccia 3D o tramite funzioni di scripting, la possibilità di visualizzazione rapida di dati attraverso finestre popup nella scena (funzione picking), e diverse funzioni di callback da OSG4Web al browser per la gestione di eventi o per l'accesso a metadata esterni.

Osg4Web è tuttora in fase di implementazione, e la roadmap per i prossimi mesi prevede l'aggiunta di ulteriori elementi, quali la ricostruzione del codice nell'ottica di realizzare un vero ambiente di sviluppo portabile ed indipendente dal core sviluppato, l'ottimizzazione del data transfer e del catching su disco fisso, l'implementazione di oggetti 3D avanzati come avatar e relative interazioni, la fruibilità di file multimediali, l'implementazione di meccanismi di software updating attraverso il web, la possibilità di collegamento degli oggetti a database esterni con pubblicazione delle informazioni nell'area 3D, la generazione di livelli 3D multipli attraverso informazioni ottenute da database spaziali e l'implementazione di strumenti per effetti di scena altamente realistici. 
Il sito web del laboratorio dedicato all'Open Source per i Beni Culturali consente di approfondire ulteriormente l'argomento, scaricare gli eseguibili del software aperto realizzato, consultare bibliografia specializzata e tutorial e infine accedere ai progetti che utilizzano i pacchetti sviluppati (http://www. vhlab.itabc.cnr.it/openheritage/).

I principali progetti su cui il sistema è stato progettato e sviluppato, secondo vari gradi di completezza, riguardano: la ricostruzione virtuale del paesaggio archeologico del Parco dell'Appia Antica a Roma (http://www.appia.itabc.cnr. it), il progetto di realizzazione del portale del Distretto Archeologico della Provincia di Salerno (http://www.distrettoarcheologicosalerno.it) e infine il progetto di interpretazione e ricostruzione del paesaggio romano della Via Flaminia Antica (http://www.vhlab.itabc.cnr.it/flaminia).

\section{Ringraziamenti}

Il VHLab del CNR ITABC, diretto da Maurizio Forte, si avvale del lavoro di un'equipe interdisciplinare composta da Carlo Camporesi e Claudio Rufa (programmatori), Nicolò Dell'Unto, Fabrizio Galeazzi, Augusto Palombini, Sofia Pescarin, Valentina Vassallo (archeologi), Marco Di Ioia, Alessia Moro, Lola Vico (architetti); Bartolomeo Trabassi (tecnico audio-video); Eva Pietroni (conservatrice BBCC). Per il CINECA Visit Lab collaborano alla realizzazione di OSG4web: Luigi Calori, Silvano Imboden e Tiziano Diamanti.

\section{Appendice (2013)}

OSG4Web è un progetto tuttora in sviluppo, ma le release create per i progetti qui trattati sono attualmente obsolete. Il principio di funzionamento e parti del codice della versione qui presentata sono elementi integranti di successivi sviluppi che hanno animato la nuova release del plug-in realizzata da ITABCCNR in collaborazione con il CINECA. La versione più recente del software attualmente disponibile è quella implementata nel progetto Aquae Patavinae (http://www.aquaepatavinae.lettere.unipd.it ultimo accesso: gennaio 2013). Per quanto riguarda i link citati nel testo, ove non diversamente specificato, l'ultimo accesso è relativo al gennaio 2013.

\section{Notes}

† CINECA - Bologna. calori@cineca.it

* Virtual Heritage Lab, Istituto per le Tecnologie Applicate ai Beni Culturali, CNR - Roma http://www.vhlab.itabc.cnr.it, carlo.camporesi@itabc.cnr.it, augusto.palombini@itabc.cnr.it, sofia.pescarin@itabc.cnr.it 


\section{Riferimenti bibliografici}

Akenine-Möller, T. e E. Haines (2002). Real-Time Rendering. $2^{\text {a }}$ ed. Massachussets US: A K Peters Natick.

Calori, L., C. Camporesi, M. Forte et al. (2005). «Interactive Landscapes reconstruction: a Web 2D and 3D Open Source solution». In: 6th International Symposium on Virtual Reality, Archeology and Cultural Heritage; 3rd Eurographics Workshop on Graphics and Cultural Heritage. ISTI CNR.

Calori, L., C. Camporesi, A. Palombini et al. (2006). «Sharing interpretation: the challenge of Open Source web approach». In: Proceedings of the Second International Conference on Remote Sensing in Archaeology. A cura di S. Campana e M. Forte. BAR International 1568. CNR Roma.

Camporesi, C, A. Palombini e A. Pescarin (2007). «Revolution OS in archeologia: esempi di interfacce web per l'archeologia del paesaggio». In: Atti del I Workshop Open Source, Free Software e open Format nei processi di ricerca archeologici. A cura di R. Bagnara e G. Macchi Janica.

Kuehne, B. e P. Martz (2007). OpenSceneGraph Reference Manual v.2.0. The OpenSceneGraph programming series. Blue Newt Software.

Pescarin S. (2006). «Open source in archeologia: nuove prospettive per la ricerca». In: Archaeologia e Calcolatori 17, pp. 137-155.

Pescarin, S. e L. Calori (2005). «Verso il VR-WebGIS. Il caso del Distretto Culturale della Valle dell'Esaro: un sistema open-source per le risorse culturali, turistiche e ambientali». In: MondoGIS 51, pp. 54-60.

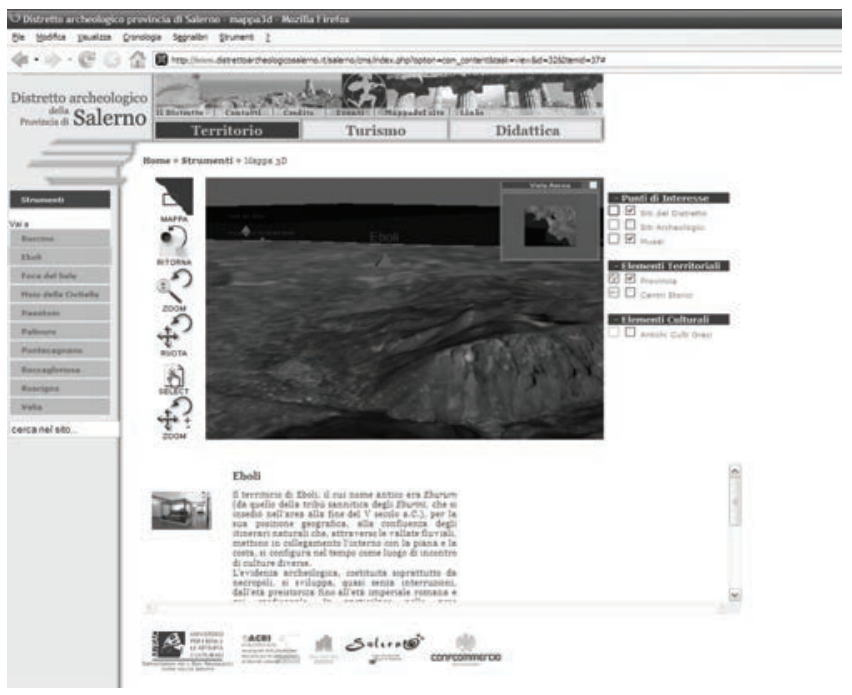

Figura 1: Osg4web: interfaccia di navigazione nel WebGis3D (dal sito del distretto archeologico di Salerno: http://www.distrettoarcheologicosalerno. it): navigazione nel paesaggio con mappa generale di riferimento. 


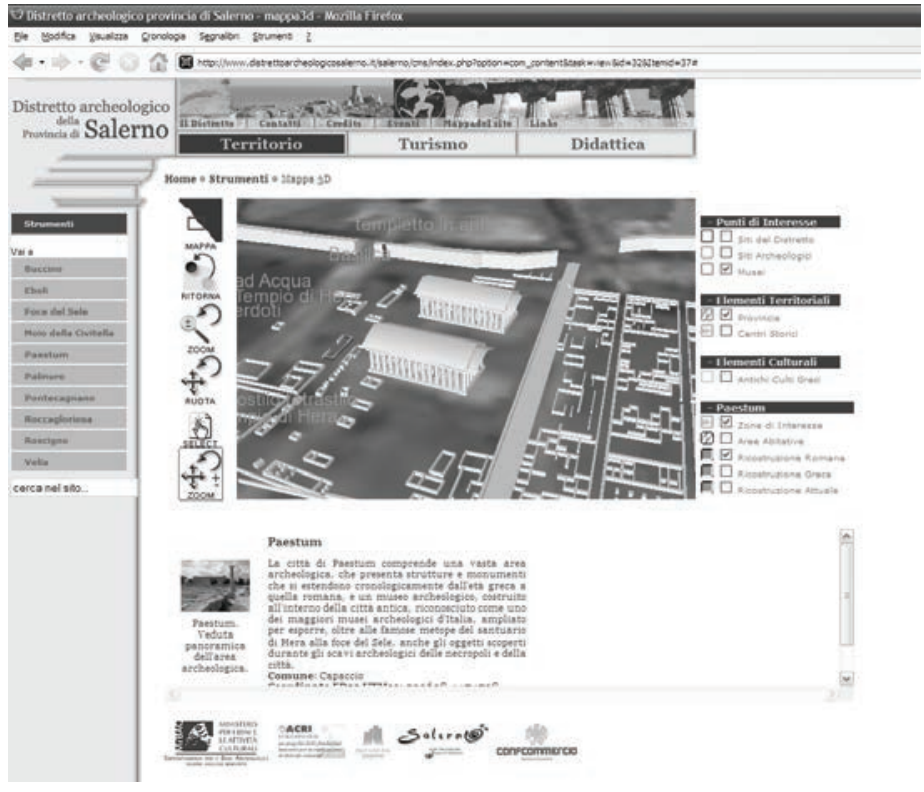

Figura 2: Osg4web: interfaccia di navigazione nel WebGis3D (dal sito del distretto archeologico di Salerno: http://www.distrettoarcheologicosalerno. it): dettaglio della ricostruzione 3D dell'area archeologica di Paestum. 